\title{
Dual-Energy X-ray Absorptiometry (DEXA) Medical Imaging Services in Zambia
}

\author{
Ernest Chanda ${ }^{1 *}$, Osward Bwanga $^{2}$, Roy Mulenga ${ }^{3}$ \\ ${ }^{\mathrm{T}}$ Diploma in Radiography, BSc Radiography, MSc Radiography, Fellowship in Radiotherapy, Postgraduate Diploma in \\ Teaching Methodology, Cancer Diseases Hospital and Lusaka Apex Medical University, Lusaka, Zambia \\ ${ }^{2}$ Diploma in Radiography, BSc Radiography, MSc Advanced Practice (Radiography), MSc Health Professions \\ Education, PhD, Midlands University Hospital Tullamore, Radiology Department, Co. Offaly, Ireland \\ ${ }^{3}$ Diploma in Radiography, University Teaching Hospital (UTH), Radiology Department, Lusaka, Zambia
}

*Corresponding Author: Ernest Chanda

Email: e.chanda75@gmail.com

\section{Article History}

Received: 24.12.2021

Accepted: 03.02.2022

Published: 09.02.2022

\begin{abstract}
Dual-energy X-ray absorptiometry (DEXA) is a medical imaging technique that uses low radiation for the diagnosis of osteoporosis. Millions of people are suffering from osteoporosis globally. The disease causes the bones to lose their strength and become more fragile and susceptible to fractures. The common risk factors of developing osteoporosis include aging, antiretroviral therapy (ARV) medication, and long-term corticosteroid medication. The morbidity and mortality of the disease arises from the associated fractures and medical complications. Although DEXA imaging services are available in the country, they remain underutilised. This article aims at bringing awareness and reviewing the DEXA imaging services in Zambia. The review found a lack of data on the prevalence of osteoporosis in Zambia. To improve the DEXA imaging services, research is required on epidemiology of the disease, associated fractures and projected costs incurred due to hospitalisation of patients.
\end{abstract}

Keywords: Dual-energy x-ray absorptiometry (DEXA), Osteoporosis, Radiographer, Zambia.

\section{INTRODUCTION}

The use of imaging in medicine for the diagnosis and treatment of diseases and injuries has increased since the discovery of X-radiation in the $19^{\text {th }}$ century. The 1970 's witnessed the rapid development in technology which resulted in discoveries of different imaging modalities such as computed tomography (CT), ultrasonography (US), and magnetic resource imaging (MRI) [1]. These medical imaging services are available in Zambia and are well-known to healthcare professionals and members of the public. However, anecdotal evidence shows that DEXA is little-known to both healthcare professionals and members of the public. DEXA is a medical imaging technique that uses low radiation for the diagnosis of osteoporosis, estimation of fracture risks, and monitoring of patients undergoing osteoporosis treatment [2]. This medical imaging examination can also demonstrate fractures.

The discovery of DEXA is accredited to John Cameron and James Sorenson who introduced technology for measuring bone mineral density (BMD) in 1963 [3]. However, the technology was ignored until 1987 when DEXA imaging was fully introduced in medicine [4]. The increase in its use and capabilities has resulted in the availability of imaging modality across the globe [3]. In Zambia, DEXA imaging services became available in 2018. People taking ARV medication, patients on long use of corticosteroid medication and the aging population are prone to osteoporosis and are potential beneficiaries of the DEXA imaging services in Zambia [5, 6]. Other indications of DEXA are discussed later.

Copyright (C) 2022 The Author(s): This is an open-access article distributed under the terms of the Creative Commons Attribution 4.0 International License (CC BY-NC 4.0) which permits unrestricted use, distribution, and reproduction in any medium for non-commercial use provided the original author and source are credited. 


\section{Zambian healthcare system}

Zambia is a landlocked country in the southern part of the Sub-Saharan African continent. It has a total area of approximately 752,614 square kilometres [7]. Zambia is located south of the Democratic Republic of Congo (DRC) and east of Angola. Other countries that share borders include Zimbabwe, Botswana, and Namibia in the south, Tanzania to the northeast, Malawi, and Mozambique in the east. There are 10 provinces in Zambia: Western, Southern, Lusaka, Central, Copperbelt, North-western, Luapula, Northern, Muchinga, and Eastern (Figure 1).

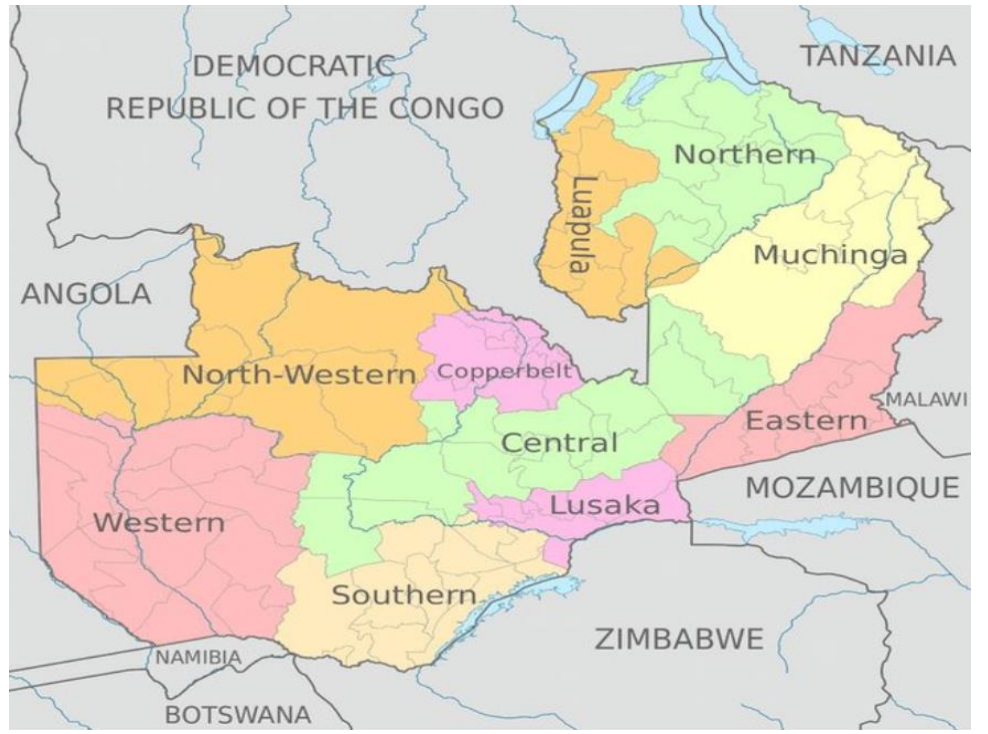

Fig-1: Map of Zambia showing provinces and neighbouring countries

The current estimated population of Zambia is 18 million, with males representing $50.1 \%$ and female $49.9 \%$ [8]. In the context of this review, knowing this ratio is important because osteoporosis is three times more common in women than in men [3]. This is due to lower peak bone mass and hormonal changes that occur at menopause in women [2,9]. It is estimated that $2.5 \%$ of the Zambian population is above the age of 65 [8]. The development of osteoporotic changes increases with the age of an individual [2]. According to the World Health Organisation (WHO) [6], the number of osteoporotic fractures increases more than 3 -fold after the age of 50.

In Zambia, the Ministry of Health $(\mathrm{MOH})$ coordinate the healthcare in the country through its headquarters in Lusaka, Provincial Health Offices [ $\mathrm{N}=10]$ at provincial level and District Health Officers [N=105] at district level [10]. Although DEXA imaging services are available in the country, literature search found no official clinical guidelines and public awareness programmes regarding the prevention, diagnosis, and management of osteoporosis. There was also no published research found on the epidemiology of the disease, associated fractures and projected costs incurred due to patient hospitalisations.

\section{Osteoporosis and global healthcare impact}

Osteoporosis is a disease in which the density and quality of bones are reduced, leading to weakness of bones and increased risks of fractures [11]. It can develop slowly over years and the first sign of the disease may be when a minor injury results in a fracture, most commonly in the wrist, hips, or spine [2]. The risk factors for developing osteoporosis include a family history of the disease, increase in age, gender, low calcium intake, long-term use of corticosteroid medication, and ARVs medication [6, 12]. The risks also depend on the race, with Whites and Asians having higher chances of developing osteoporosis than other races [13, 14].

Other indications for DEXA include patients with a history of arthritis, women who attained early menopause, underwent bilateral oophorectomy before the age of forty-five years without undergoing hormonal replacement therapy [15]. Post-menopausal women with a lifestyle of heavy drinking and smoking, as well as women with enormous breaks in between menses amounting to more than a year are also prone to osteoporosis [2, 15]. Bonnick [15] adds that people who experience a bone fracture after a negligible fall and those with a family history of hip fractures are candidates to undergo a DEXA scan. Patients with endocrine disorders is another reason for requesting for DEXA scan [16]. In endocrine disorders patients may have insignificant change in BMD or it can be regular, fractures in these cases are associated with a reduction of bone quality than BMD. Therefore, DEXA cannot be used to indicate the risk of a fracture. However, it is indicated in vertebral fracture assessment which is easily picked up fractures in areas like the lateral thoracolumbar region [2, 15]. DEXA scanner uses reduced dose radiation as compared to the conventional X-rays 
making it more precise in diagnosing fractures in these areas. As heightened, there are many indications of DEXA imaging examination.

Osteoporosis is a global health problem; this disease affects more than 75 million people in Europe, Japan, and the USA [13]. There is a paucity of data on the number of people with osteoporosis in Africa. According to the International Osteoporosis Foundation (IOF) [17], in Kenya, osteoporosis in women above 50 stands at $24.5 \%$, and in Egypt, $28.4 \%$ and $21.9 \%$ of postmenopausal women and men respectively have osteoporosis. In South Africa, it is estimated that 1.4 million females and 0.6 million males aged over 50 are suffering from osteoporosis [13, 17]. More research is required to know the prevalence of the disease in Africa.

The morbidity and mortality of osteoporosis arise from the associated fractures [9, 11]. It is estimated that about 2.3 million fractures in Europe and the USA are caused by osteoporosis annually [13]. There is also a lack of data on the number of fractures caused by osteoporosis in Africa, including Zambia. However, a discussion with an orthopaedic consultant surgeon at the University Teaching Hospital (UTH) of Lusaka, revealed that 25\% of fractures in Zambia are caused by osteoporosis. This disease is a global health concern because it results some people to be bedridden with secondary medical complications that need hospitalisation [9]. The disability due to osteoporosis is greater than that caused by chronic non-communicable diseases, such as rheumatoid arthritis, asthma, and high blood pressure [13].

Osteoporosis was considered as an under-recognised disease and considered to be an inevitable consequence of aging [6]. This has resulted in non-inclusion of the diagnosis and treatment of osteoporosis in medical school curriculums in most of African countries [17]. The audit conducted by the International Osteoporosis Foundation (IOF) [17] also found non-availability of official clinical guidelines in Kenya regarding osteoporosis diagnosis, prevention, and treatment. However, the perceptions amongst healthcare professionals and policymakers in most countries have now changed due to the high burden of the disease and its costs to society and healthcare facilities, as well as the adverse effects on millions of patients worldwide $[6,13]$.

\section{DEXA imaging workforce in Zambia}

The DEXA imaging workforce in Zambia consists of three imaging professionals: radiography technologists, radiographers, and radiologists. A radiography technologist holds a diploma in diagnostic radiography, whilst a radiographer holds a degree in diagnostic radiography. Both are trained to produce high-quality images using radiation to assist medical practitioners in the diagnosis of diseases and injuries. Amongst the 919 radiography technologists and radiographers working in Zambia [7], only nine are trained in DEXA. On the other hand, a radiologist is a medical doctor specailised in diagnosing and treating diseases and injuries using imaging. One of their roles is to report on radiographic images, including DEXA images. Nine radiologists are working in public hospitals in the country [18]. The critical shortage of radiologists is negatively affecting the delivery of imaging services in the country as most of the radiographic images go unreported. In most cases, referring clinicians have taken up the responsibility of interpreting of radiographic images. Radiologists only reports on specialised imaging examinations, such as CT, MRI, and nuclear medicine.

\section{Hospitals offering DEXA imaging services in Zambia}

Two hospitals are offering DEXA imaging services in the country: University Teaching Hospital (UTH) and Medland Hospital of Lusaka.

\section{University Teaching Hospital (UTH)}

The University Teaching Hospital (UTH) is the largest public hospital in the country, with a bed capacity of 1,800. It is in Lusaka, the capital city, and offers a full range of primary, secondary, and tertiary health, and medical services. UTH is a centre for specialist referrals from all hospitals across the country. The hospital is also the principal clinical training centre for radiography, medical, nursing, and other healthcare students. The main radiology department is equipped with a range of different imaging modalities: conventional radiography, fluoroscopy, US, CT, nuclear medicine, catheterisation laboratory (Cath Lab) and DEXA. The DEXA equipment was donated by the Center for Infectious Disease Research in Zambia (CIDRZ), with support from the United States of America National Institute of Health [5]. It was commissioned on $20^{\text {th }}$ august 2018. Figures 2 and 3 show the DEXA scanner at UTH. 


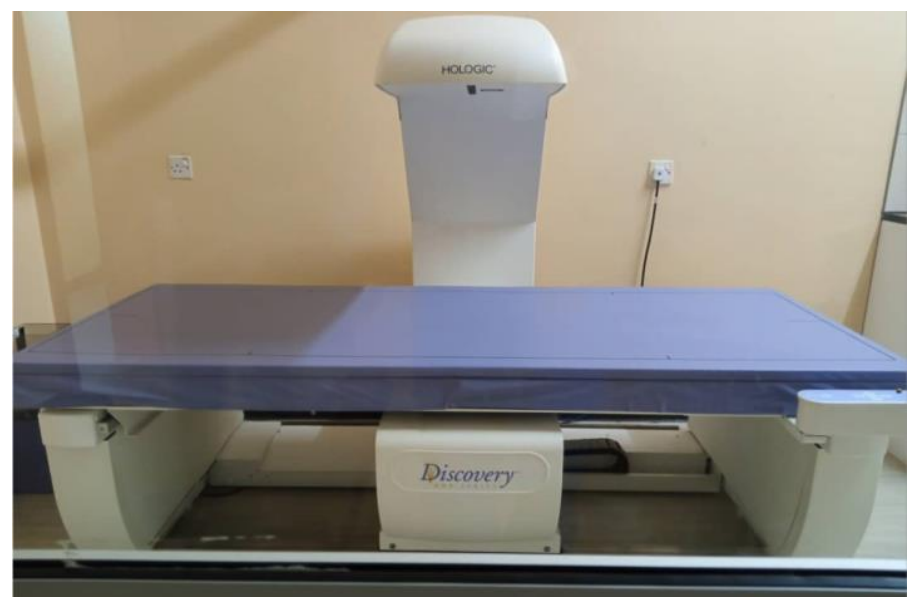

Fig-2: DEXA scanner (frontal view)

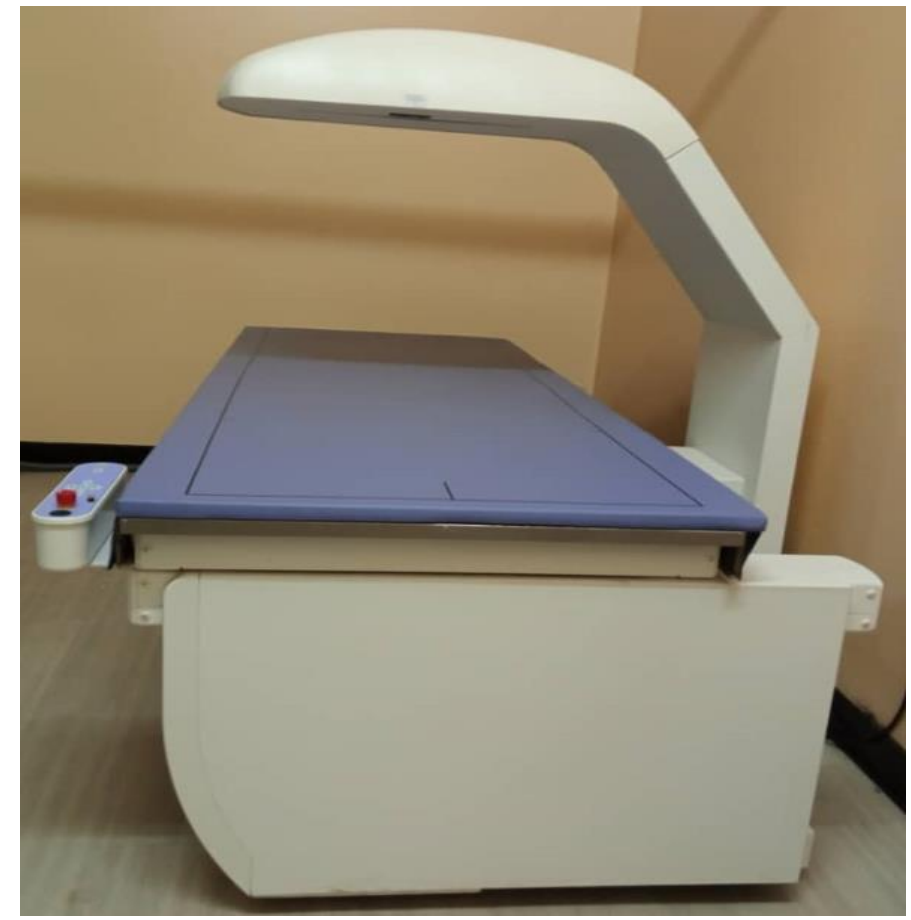

Fig-3: DEXA scanner (side view)

The radiology department is staffed with 36 radiography technologists, 15 radiographers, and three radiologists. Amongst the radiography technologists and radiographers, six are trained in DEXA. The main clinicians who refer patients for DEXA include oncologists, orthopaedic surgeons, physicians, and physiotherapists. Since the inception of the imaging service, the youngest patient has been 26 years and the oldest 77 years. Over $90 \%$ of the patient age range between 42 and 77 years. The common examinations performed include the spine, hip, forearm, and whole-body scans. In 2019, a total of 89 patients were scanned: 35 females and 54 males. This number is very low when compared to the population of Zambia. This may be due to a lack of awareness amongst the healthcare professionals who refer patients for imaging examinations.

\section{Medland Hospital}

Medland Hospital is a private health facility located in Lusaka with a bed capacity of 78. There are two radiographers, one radiography technologist, and two radiologists working in the radiology department (one permanent and another based in India who reports electronically). The hospital offers the following imaging services: conventional radiography, US, CT, mammography, MRI, and DEXA. The DEXA equipment was installed in July 2019. All radiographers and a radiography technologist perform DEXA imaging examinations.

\section{DEXA image interpretation and reporting}

The DEXA reports are automated with the machine producing the diagnostic information and generating recommendations. The interpretation is performed by the radiologist or referring clinician. For this reason, clinicians 
involved in the management of osteoporosis patients must understand the auto-generated DEXA results. This is to enable them to estimate the patients' risk of developing an osteoporotic associated fracture [19]. The anatomical sites scanned are the lumbar spine, hip (neck of femur), or forearm (wrist). These anatomical sites are prone to osteoporotic fractures $[2,15]$. Two scores are used in the interpretation of DEXA results known as the $\mathrm{T}$ and $\mathrm{Z}$ scores $[2,15]$. The $\mathrm{T}$-score represents the normal reference percentage which is derived from the BMD of a healthy 30-year-old, the age where the bones are at their strongest point [15]. This helps to indicate how much lower or higher the bone density is in the patient under study [15]. The lower the score the weaker the bones and the higher the risk of osteoporotic-related fractures. Table I shows the 3 categories on a scale that helps in the interpretation of the DEXA results.

Table-I: Three categories of interpreting DEXA results

\begin{tabular}{|l|l|l|}
\hline & T-score & Interpretation \\
\hline 1 & T-score of -1.0 or above & Normal bone density \\
\hline 2 & T-score between -1.0 and -2.5 & Low bone density or osteopenia \\
\hline 3 & T-score of -2.5 or lower & Osteoporosis \\
\hline
\end{tabular}

On the other hand, the Z-score (percentage of the same age) compares the bone density to a normal score for a person with the same body size and age $[2,15]$. With the critical shortage of radiologists in Zambia, this is one area where radiographers can extend their role by interpreting and reporting on DEXA imaging examinations.

\section{Education and training for the DEXA radiography workforce}

To ensure DEXA imaging services are safe, high quality and effective, it is important that imaging professionals are appropriately trained [20]. It is a statutory requirement under the ionising radiation protection act of 2011 of the Republic of Zambia [21] that all those undertaking radiological examinations must be appropriately trained. DEXA imaging modality has been integrated in the degree in diagnostic radiography programmes for radiographers offered by the Lusaka Apex Medical University (LAMU) and University of Zambia (UNZA). Although this can provide adequate education and practical skills, the College of Radiographers of the United Kingdom (UK) [20] recommends specialised DEXA training.

The literature search revealed two main types of training for radiography workforce: application specialists training and formal course. Specific equipment training can be provided to radiography technologists and radiographers by application specialists. This was the type of training provided to radiography technologists and radiographers at UTH and Medland Hospital. Formal courses are also provided by academic institutions. For example, in the Republic of Ireland, the University College Dublin (UCD) [22], offers a four-month postgraduate certificate in DEXA imaging. In the UK, the University of Derby [23], also provides a postgraduate certificate in bone densitometry as well as image interpretation and reporting. These formal courses provide an understanding of the operational and technical skills required to produce optimal scan results as well as image interpretation and reporting. However, a review conducted by Bwanga and others [24] on the readiness of Zambia in establishing postgraduate training in specialised diagnostic imaging did not include DEXA. It should be mentioned that DEXA should also be consider for postgraduate education and training in Zambia.

\section{Challenges of DEXA imaging services in Zambia}

The main challenges of DEXA imaging services in Zambia can be grouped under three themes (Table II).

Table-II: Challenges for DEXA imaging services in Zambia

\begin{tabular}{|l|l|}
\hline No & Challenge \\
\hline 1 & Lack of awareness about the availability of DEXA imaging services in the country \\
\hline 2 & Limitation of the available DEXA imaging equipment \\
\hline 3 & Non-inclusion of DEXA imaging in the diploma in radiography curriculum \\
\hline
\end{tabular}

There is a lack of awareness amongst healthcare professionals about the availability of DEXA imaging services in the county. This can be seen with the low number of patients being referred for DEXA examinations. This observation concur with the research conducted in Egypt by El Badawy and others [25], where the awareness levels about the osteoporosis and imaging methods amongst healthcare professionals were found to be below average (47\%). An audit conducted in Kenya also found awareness levels of healthcare professionals about the osteoporosis to be low, except for orthopaedic surgeons who frequently referred patients for DEXA imaging [17].

There are two types of DEXA scanners: central units for spine, whole body and hips and peripheral units for extremities such as forearm and legs. UTH has a central DEXA equipment, whilst the Medland Hospital has the peripheral one. This limitation of equipment is a challenge, UTH sometimes receive patients for extremity examinations. In this case, the technique is modified, or patients referred to Medland Hospital who has equipment for extremities. 
The DEXA imaging modality has been integrated in the two degree in diagnostic radiography programmes offered by LAMU and UNZA. However, it is excluded in the diploma in diagnostic radiography programme for radiography technologists offered by Evelyn Hone College (EHC). This may be attributed due to the history of osteoporosis which was an under-recognised disease [6]. Radiography students are also rarely exposed to DEXA during their clinical placements due to limited patients seen at UTH, the main radiography clinical training site. This has kept this imaging modality a little-known and not an obvious option for radiography technologists and radiographers to consider postgraduate specialisation.

\section{RECOMMENDATIONS}

The following recommendations are made to improve DEXA imaging services in Zambia:

- To establish DEXA imaging awareness programmes for medical practitioners through clinical meetings.

- To consider procuring DEXA equipment with both central imaging and extremities capabilities when expanding the services.

- To include DEXA imaging modality in the radiography diploma programme at EHC to impart radiography technologists with appropriate knowledge and skills. This is against the background that EHC contributes more than three quarters of radiography workforce in the country [26].

\section{CONCLUSION}

This review has provided an overview of DEXA imaging services in Zambia. However, there was a lack of data on the prevalence of osteoporosis in the country. Research is required to provide baseline information that can be used to advocate for more DEXA imaging equipment and expansion of services to all provinces of Zambia.

\section{ACKNOWLEDGEMENTS}

Special thanks go to Professor Godfrey Azangwe for his insights and contributions during the production of the manuscript. Theresa Imenda, DEXA radiographer, Dr Dorothy Lombe and Ethel Chanda also for the support and contributions to the article.

\section{REFERENCES}

1. Carter, P.H., Peterson, A.M., Thornton, M.L., Hyatt, A.P., Milne, A. \& Pirrie, J.R. (1994). Chesneys' equipment for student radiographers. $4^{\text {th }}$ ed. Oxford: Blackwell Publishing.

2. El Maghraoui, A. (2012). Dual-energy x-ray absorptiometry. Rijeka: InTech.

3. Lander, A.S. (2011). DEXA evolution. From https://www.bioclinica.com/assets /Uploads/DXAEvolution_Feb2011.pdf (accessed on 10/07/2020).

4. International Society for Clinical Densitometry (2020). History of DEXA. From https://www.iscd.org/about/history/ (accessed on 10/07/2020).

5. Center for Infectious Disease Research in Zambia. (2018). CIDRZ donates DEXA machine to UTH, first ever machine in Zambia. From http://www.cidrz.org/ cidrz-donates-dexa-machine-to-uth-first-ever-machine-in-zambia/ (accessed on 25/07/2020).

6. World Health Organisation. (2007). WHO scientific group on the assessment of osteoporosis at primary health care level. Geneva: WHO.

7. Mbewe, C., Chanda-Kapata, P., Sunkutu-Sichizya, V., Lambwe, N., Yakovlyeva, N., Chirwa, M., Ayele, B., \& Pitcher, R. D. (2020). An audit of licenced Zambian diagnostic imaging equipment and personnel. Pan African Medical Journal. 1-14.

8. Country meters. (2020). Zambia population. From https://countrymeters. Info /en/ Zambia (accessed on 20/07/2020).

9. World Health Organisation. (2003). Prevention and management of osteoporosis. Geneva: WHO.

10. Ministry of Health. (2017). National health strategic plan 2017-2021. Lusaka: The Ministry of Health.

11. National Osteoporosis Foundation of South Africa. (2020). Mission and vision. From https://osteoporosis.org.za/about-us/mission-vision/(accessed on 20/07/2020).

12. Kruger, M. J., \& Nell, T., A. (2017). Bone mineral density in people living with HIV: a narrative review of the literature. AIDS Res \& Therapy, 14(1); 35-47.

13. International Osteoporosis Foundation. (2017). Facts and statistics. From https://www.iofbonehealth.org/factsstatistics/(accessed on 18/07/2020).

14. Gheita, T. A., \& Hammam, N. (2018). Epidemiology and awareness of osteoporosis: a viewpoint from the Middle East and North Africa. International Journal of Clinical Rheumatology, 13(3); 134-147.

15. Bonnick, S.L. (2010). Bone densitometry in clinical practice-application and interpretation. $3^{\text {rd }}$ Ed. Denton, TX: Humuna Press.

16. Vainicher, C. E., Falchetti, A., Gennari, L., Cairoli, E., Bertoldo, F., Vescini, F., Scillitani, A., \& Chiodini, I. (2019). Diagnosis of endocrine disease: evaluation of bone fragility in endocrine disorders. European journal of 
endocrinology.180-186.

17. International Osteoporosis Foundation (2020). The Middle East and Africa regional audit- Epidemiology, costs \& burden of osteoporosis in 2011. From https:// www.iofbonehealth.org/data-publications/regional-audits/middle-eastafrica-audit (accessed on 18/08/2020).

18. Bwanga, O., Chanda, E., Kafwimbi, S., \& Sichone, J. (2021). Opinions of Zambian radiographers on extending their role in interpretation and reporting on general radiographic images: a cross-sectional survey. Medical Journal of Zambia, 48(3), 212 - 220.

19. Choplin, R.H., Lenchik, L., \& Wuertzer, S. (2014). A practical approach to interpretation of dual energy x-ray absorptiometry (DXA) for assessment on bone density. Curr Radiol Rep. 2:48

20. College of Radiographers. (2018). The role of the radiographer in DXA and osteoporosis services. London: College of Radiographers.

21. Republic of Zambia. (2011). Ionising radiation protection act of 2011 (Amended). Lusaka: Government Printers.

22. University College Dublin. (2020). Professional certificate dual energy $\mathrm{x}$-ray absorptiometry. From https://www.ucd.ie/medicine/studywithus/ graduatestudies /radiography/fullportfolio/professionalcertificatedualenergyx-rayabsorptiometry/ (accessed on 25/07/2020).

23. University of Derby (2020). Bone densitometry reporting. From https://www. derby.ac.uk/postgraduate/radiographycourses/bone-densitometry-reporting-pg-cert//(accessed on 25/07/2020).

24. Bwanga, O., Mwansa, E., Sichone, J., \& Kafwimbi, S. (2020). Establishment of postgraduate education and training in the specialised areas of diagnostic imaging in Zambia. African Journal of Health, Nursing and Midwifery, 3(4); 55-64.

25. El Badawy, A.A., Sharkawy, G.F., Fahmy, H.H., Demitry, S.R., Sherif, N.A., \& El Badawy. (2009). Awareness about osteoporosis among a sample of women and healthcare providers in Zagazig District Egypt. Egyptian Osteoporosis Prevention Society Conference.

26. Bwanga, O. (2020). Developing a framework strategy for supporting radiographers in the clinical supervision of radiography students in Zambia: a mixed methods study. Ethiopian Journal of Health Sciences, 30(6); 971-980. 